\title{
HSV-TK-Transduced Donor Lymphocytes
}

National Cancer Institute

\section{Source}

National Cancer Institute. HSV-TK-Transduced Donor Lymphocytes. NCI Thesaurus.

Code C67047.

A preparation of donor lymphocytes transduced with the suicide gene herpes simplex virus thymidine kinase (HSV-TK) with potential immunomodulating activity.

Administration of HSV-T K-transduced lymphocytes after T cell-depleted allogeneic stem cell transplantation allows an early controllable immune reconstitution, which takes advantage of the antitumor effect of donor lymphocytes and helps to mitigate the risk of post-transplant opportunistic infection. To control graft-versus-host disease (GvHD) due to donor lymphocyte infusion, HSV-TK-transduced donor lymphocytes are selectively eliminated by administration of the antiviral agent ganciclovir. Ganciclovir, a prodrug, is readily phosphorylated by the suicide gene HSV-TK within HSV-TK-transduced lymphocytes to its monophosphate form and, subsequently, converted into its active triphosphate form, which specifically kills HSV-TK- transduced donor lymphocytes. 